\title{
openheart A prognostic scoring system for arm exercise stress testing
}

\author{
Yan Xie, ${ }^{1}$ Hong Xian, ${ }^{1,2}$ Pooja Chandiramani, ${ }^{3}$ Emily Bainter, ${ }^{4}$ Leping Wan, ${ }^{2}$ \\ Wade $\mathrm{H}$ Martin III
}

To cite: Xie $\mathrm{Y}, \mathrm{Xian} \mathrm{H}$, Chandiramani $\mathrm{P}$, et al. A prognostic scoring system for arm exercise stress testing. Open Heart 2016;3: e000333. doi:10.1136/ openhrt-2015-000333

Received 1 September 2015 Revised 29 October 2015 Accepted 3 December 2015

\section{(a) CrossMark}

${ }^{1}$ From the Clinical Epidemiology Center of the St. Louis Veterans Administration Medical Center, College of Public Health and Social Justice, St. Louis University, St. Louis, Missouri, USA ${ }^{2}$ Department of Biostatistics, College of Public Health and Social Justice, St. Louis University, St. Louis, Missouri, USA

${ }^{3}$ Department of

Epidemiology, College of Public Health and Social Justice, St. Louis University, St. Louis, Missouri, USA ${ }^{4}$ Department of Internal Medicine, Washington University School of Medicine, St. Louis, Missouri, USA

${ }^{5}$ Division of Cardiology, Department of Internal Medicine, St. Louis Veterans Administration Medical Center and Washington University School of Medicine, St. Louis, Missouri, USA

Correspondence to Dr Wade H Martin III; Wade.Martin@va.gov

\section{ABSTRACT}

Objective: Arm exercise stress testing may be an equivalent or better predictor of mortality outcome than pharmacological stress imaging for the $\geq 50 \%$ for patients unable to perform leg exercise. Thus, our objective was to develop an arm exercise ECG stress test scoring system, analogous to the Duke Treadmill Score, for predicting outcome in these individuals.

Methods: In this retrospective observational cohort study, arm exercise ECG stress tests were performed in 443 consecutive veterans aged 64.1 (11.1) years. (mean (SD)) between 1997 and 2002. From multivariate Cox models, arm exercise scores were developed for prediction of 5-year and 12-year allcause and cardiovascular mortality and 5-year cardiovascular mortality or myocardial infarction (MI).

Results: Arm exercise capacity in resting metabolic equivalents (METs), 1 min heart rate recovery (HRR) and ST segment depression $\geq 1 \mathrm{~mm}$ were the stress test variables independently associated with all-cause and cardiovascular mortality by step-wise Cox analysis (all $p<0.01)$. A score based on the relation HRR (bpm) $+7.3 \times$ METs $-10.5 \times$ ST depression ( $0=$ no; $1=y e s)$ prognosticated 5 -year cardiovascular mortality with a C-statistic of 0.81 before and 0.88 after adjustment for significant demographic and clinical covariates. Arm exercise scores for the other outcome end points yielded C-statistic values of $0.77-0.79$ before and 0.82-0.86 after adjustment for significant covariates versus $0.64-0.72$ for best fit pharmacological myocardial perfusion imaging models in a cohort of 1730 veterans who were evaluated over the same time period.

Conclusions: Arm exercise scores, analogous to the Duke Treadmill Score, have good power for prediction of mortality or MI in patients who cannot perform leg exercise.

\section{INTRODUCTION}

The Duke Treadmill Score has been well validated and widely used for many years as a powerful prognostic marker in patients being evaluated for coronary artery disease. ${ }^{1-3}$ However, in recent years at least $50 \%$ of patients referred for stress testing are unable to perform adequate treadmill exercise because of lower extremity or other disabilities and pharmacological evaluations are

\section{KEY QUESTIONS}

What is already known about this subject?

- No scoring system exists for outcome prediction from arm exercise stress test results in the $\geq 50 \%$ of patients unable to perform leg exercise.

What does this study add?

- We developed a scoring system, analogous to the Duke Treadmill Score, for arm exercise stress testing.

How might this impact on clinical practice?

- With this scoring system, 5-year and 12-year allcause and cardiovascular mortality or myocardial infarction can be estimated from easily measured arm exercise stress test variables with comparable accuracy to the Duke Treadmill Score in patients unable to perform leg exercise.

employed as a surrogate form of stress, ${ }^{4}$ for which the Duke Treadmill Score is not relevant. In addition, the Duke Treadmill Score does not include variables such as heart rate recovery that have been found to be highly predictive of mortality. ${ }^{5}{ }^{6}$ Although pharmacological stress tests may yield useful imaging results, they fail to provide powerful prognostic information on exercise capacity, symptomatic, physiological or ECG responses to the relevant physiological stress of exercise. We have demonstrated that arm exercise stress testing is at least equivalent and possibly a superior alternative to pharmacological evaluations ${ }^{7}$ in patients unable to perform leg exercise and is predictive of all-cause and cardiovascular mortality over 5 and 12 years ${ }^{7-9}$ and myocardial infarction (MI) at 5 years. ${ }^{10}$ However, a simple scoring system, analogous to the Duke Treadmill Score, is not currently available for arm exercise testing. Thus, the purpose of this investigation was to develop and report a simple scoring system for predicting outcome, defined in terms of 5-year and 12-year all-cause mortality, 5-year and 12-year cardiovascular mortality, and 5-year cardiovascular mortality or MI for arm exercise ECG stress testing. 


\section{METHODS}

\section{Patient characteristics and covariate information}

The arm exercise scores were derived from a patient population of 443 consecutive individuals (434 men, 9 women) aged 64.1 (11.1) years (mean (SD)) who underwent arm ergometer exercise stress tests at the St. Louis Veterans Administration Medical Center (STL VAMC) from 1997 until 2002 and were described in an earlier publication. ${ }^{7}$ Veterans who were referred by their VA healthcare providers, were unable to perform treadmill or leg cycle ergometer exercise because of lower extremity or other disabilities, and who underwent arm exercise testing, were eligible for the study. Those having upper extremity disabilities or medical contraindications to stress testing, identified in a brief history and physical examination, were excluded. In $<3 \%$ of tests, arm exercise testing was substituted for pharmacological (dipyridamole) evaluations because of caffeinated beverage ingestion and a long travel distance to St. Louis. Arm exercise studies were converted to pharmacological evaluations in $<2 \%$ of participants because of inability to exercise for at least $1 \mathrm{~min}$. Participants were instructed to fast overnight, withhold $\beta$-adrenergic blocking agents, administer $1 / 2$ of their usual insulin dose, and take all other regular medications. The study was approved by the Institutional Review Board of the STL VAMC. All patients provided voluntary written informed consent.

\section{Arm exercise protocol}

The stress test was conducted in the seated posture with a wall-mounted electronically braked arm cycle ergometer (Angio 2000; Lode BV, Groningen, the Netherlands) at a target cadence of $60 \mathrm{rpm}$ to an end point of fatigue or symptom limitation as described previously. ${ }^{8}$ A progressive, multistage protocol designed to elicit exhaustion or symptoms within 5-12 min was used with constant work increments of $50-200 \mathrm{kpm}$, equivalent to $8.2-32.7 \mathrm{~W}$, every $2-3 \mathrm{~min}$, depending on pretest estimated exercise capacity. ${ }^{11}{ }^{12}$ After a brief clinical evaluation, a 12-lead ECG was recorded at baseline, every minute during and postexercise and continuously at peak effort. Blood pressure was obtained manually at baseline and every 2-3 min during and postexercise. Exercise capacity in METs (multiples of resting metabolic rate) was determined from the duration of effort at the peak exercise work rate using the standard relationship between oxygen uptake and cycle ergometer work rate, with 1 MET equivalent to $3.5 \mathrm{~mL} / \mathrm{kg} / \mathrm{min}$ and exercise METs $=((2 \times \mathrm{kpm})+300) /($ weight $(\mathrm{kg}) \times 3.5)) .{ }^{8}{ }^{13}$ Peak heart rate and heart rate recovery $1 \mathrm{~min}$ postexercise were calculated from the ECG. One minute heart rate recovery in beats/min was determined as the difference between peak heart rate and the heart rate $1 \mathrm{~min}$ postexercise. ${ }^{5}$ Standard criteria were used for interpretation of the exercise ECG with an abnormal tracing defined as $\geq 1 \mathrm{~mm}$ of ST segment depression, measured $80 \mathrm{~ms}$ after the J-point. ${ }^{11}$

\section{Outcome events}

Occurrence and date of death and MI, probable cause of death, demographic, and clinical characteristics of participants, medications prescribed, and coronary revascularisation procedures were ascertained by review of VA electronic medical records preceding and following stress testing until 5 November 2012 (10th anniversary of the last participant stress test). This information was supplemented by examination of scanned reports of non-VA episodes of care, telephone interviews of next of kin, and data from death certificates, the Missouri Department of Health and Senior Services, Social Security Death Index and National Death Index. Research staff were blinded to stress test findings at the time of record review. The criteria for MI were elevated creatine kinase-MB or troponin above the 99th centile in the context of typical ECG findings or symptoms. Censoring was not performed after MI or coronary revascularisation.

\section{Data analysis}

Statistically significant differences between group means were determined with $\mathrm{t}$ tests. $\chi^{2}$ Analyses were used to examine associations of categorical variables with mortality. Missing data were not imputed and analyses included only existing values. Differences were considered significant at $p<0.05$. Arm exercise variables identified in univariate analyses to be predictive of 5-year and 12-year all-cause and cardiovascular mortality and 5-year cardiovascular mortality or MI were incorporated into multivariate Cox proportional hazards models. After removal of non-significant variables from these models, scores to predict outcomes were then developed using remaining arm exercise variables in the respective multivariate models. ${ }^{12}$ Complete arm exercise data were available in 331 participants and arm exercise scores were developed from this cohort. Weights were assigned to each independently predictive exercise variable, based on their effect size coefficient for a given outcome model. ${ }^{1}{ }^{2}$ Relationships of scores and significant demographic and clinical variables with the various outcomes were also evaluated with univariate and multivariate Cox proportional hazards models. C-statistics were used to evaluate model discrimination of arm exercise scores before and after adjustment for significant demographic and clinical variables and to compare the predictive value of arm exercise scores with our recently published pharmacological MPI results. ${ }^{7}$ Kaplan-Meier curves were generated to compare rates of survival or MI among patient groups. Score cut points were selected to categorise patients into high and low-risk groups, based on sequential step-wise comparisons of adjacent Kaplan-Meier curve deciles beginning with the worst and most favourable outcome extremes, based on survival probability. The log rank test for survival analysis was employed to assess differences in Kaplan-Meier curve deciles. The logistic growth model with the smallest sum of squares was used to estimate relationships between arm exercise 
scores and survival. All analyses were performed with SAS V. $9.4 .^{14}$

\section{RESULTS}

\section{Outcome events}

There were 256 deaths (57.8\%) over mean (SD) and median (IQR) follow-up intervals of 12.0 (1.3) and 11.5 $(10.8,13.1)$ years, respectively, for survivors and 8.2 (3.4) and $9.5(4.3,11.4)$ years, respectively, for the entire cohort. Of 252 participants for whom probable cause of death could be ascertained (98.4\%), 102 were attributed to a cardiovascular aetiology $(40.4 \%)$. During follow-up, 70 participants (15.8\%) experienced MI, 39 underwent coronary artery bypass graft surgery $(8.8 \%)$ and 78 had percutaneous coronary interventions $(17.6 \%)$.

\section{Patient characteristics}

Demographic and clinical characteristics of this population and their univariate associations with all-cause and cardiovascular mortality at 5 and 12 years are shown in table 1.

Resting ECG abnormalities that may preclude reliable interpretation of the stress ECG included left bundle branch block (2.0\%), ST segment abnormalities $\geq 1 \mathrm{~mm}$ $(7.2 \%)$, and a ventricular paced rhythm (1.6\%) but an abnormal resting ECG was not associated with mortality by multivariate analysis.

Similarly, although cardiovascular medications were prescribed for a high proportion of patients in this cohort, none were related to mortality after adjustment for covariates. These medications included $\beta$ adrenergic blocking agents $(40.6 \%)$, an ACE inhibitor, receptor blocker or hydralazine $(52.8 \%)$, statin $(36.6 \%)$, platelet antagonist $(57.1 \%)$ and aldosterone antagonist (3.8\%).

\section{Arm exercise results}

Arm exercise findings with univariate significance are shown in table 1 above and multivariate analyses of significant univariate arm exercise results reported in tables 2 and 3 below. Arm exercise capacity in METs, $1 \mathrm{~min}$ heart rate recovery and ST segment depression $\geq 1 \mathrm{~mm}$ were the only exercise variables independently associated with death or MI by multivariate analysis. For arm exercise capacity, the multivariate inverse relationship with 12-year all-cause mortality, expressed per SD of METs, was HR $0.57,95 \%$ CI 0.46 to 0.67 ; $\mathrm{p}<0.001$ while the corresponding relationship with 12-year cardiovascular death was HR $0.51,95 \%$ CI 0.38 to $0.68 ; \mathrm{p}<0.001$. For $1 \mathrm{~min}$ heart rate recovery, the multivariate inverse relationship with 12-year all-cause mortality, expressed per SD of heart rate recovery, was HR $0.66,95 \%$ CI 0.55 to $0.79 ; \mathrm{p}<0.001$ and for 12-year cardiovascular death was HR $0.60,95 \%$ CI 0.45 to $0.82 ; \mathrm{p}<0.01$. Arm exercise-induced ST segment depression $\geq 1 \mathrm{~mm}$ was directly associated with outcome end points as shown in tables 2 and 3 .

Neither limiting nor non-limiting angina predicted death $(p>0.05$ by multivariate analysis). Exercise-induced ventricular dysrhythmias were related to mortality by univariate analysis $(\mathrm{p}<0.01)$ but not after adjustment for covariates. Coronary revascularisation by either coronary artery bypass graft surgery or percutaneous coronary intervention was associated with lower mortality (HR $0.52,95 \%$ CI 0.38 to $0.72 ; \mathrm{p}<0.001)$. However, there was no interaction between coronary revascularisation and the inverse relationship of arm exercise capacity and heart rate recovery with death. Arm exercise-induced ST segment depression was predictive of coronary revascularisation at 5 and 12 years (HR 2.29, 95\% CI 1.25 to 4.19; $\mathrm{p}=0.007$ and HR 2.42, 95\% CI 1.55 to $3.77 ; \mathrm{p}<0.001$, respectively). Limiting angina also was associated with coronary revascularisation at 5 and 12 years (HR 7.12, 95\% CI 2.14 to $23.7 ; \mathrm{p}=0.001$ and HR $4.75,95 \%$ CI 1.46 to $15.42 ; \mathrm{p}<0.001$, respectively).

Although $\beta$-adrenergic blocking agents were withheld on the morning of testing, their use was still associated with lower resting heart rate (70 (13) vs 78 (15) bpm), peak heart rate $(115(23)$ vs $130(20) \mathrm{bpm})$ and $\delta$ (peak -resting) heart rate (45 (31) vs 52 (20) bpm) (all $\mathrm{p}<0.01$ ) but did not affect arm exercise capacity in METs, heart rate recovery or ST segment depression. In addition, multivariate Cox proportional hazards analyses revealed no significant interaction between treatment with $\beta$ blockade and prediction of mortality with arm exercise capacity in METs, 1 min heart rate recovery, and ST segment depression, the components of the arm exercise score.

\section{Arm exercise scores}

For arm exercise variables, data were available for the following number and percentage of participants: METs $(\mathrm{n}=440 ; 99.3 \%)$, peak heart rate $(\mathrm{n}=443 ; 100 \%)$, peak systolic blood pressure $(\mathrm{n}=433 ; 97.5 \%), 1$ min heart rate recovery $(n=340 ; 76.7 \%)$, angina $(n=443 ; 100 \%)$, ventricular dysrhythmias $(\mathrm{n}=443 ; 100 \%)$ and an interpretable ST segment response $(\mathrm{n}=427 ; 96.4 \%)$. Arm exercise scores were developed from complete data in 331 participants. Multivariate HRs and 95\% CIs for arm exercise scores and significant univariate demographic and clinical associations with the respective outcomes in Cox proportional hazards models are shown in table 4 .

In figure 1, Kaplan-Meier curves for high and low-risk groups, based on the displayed arm exercise score cut points, are depicted for 5-year all-cause death (A), 5-year cardiovascular death (B), 12-year all-cause death (C) and 12-year cardiovascular death (D). Arm exercise scores and C-statistic values for prediction of outcome events before and after adjustment for significant demographic and clinical covariates are presented in table 5 .

To permit estimation of survival rates of individual patients with a given arm exercise score, continuous relationships between corresponding arm exercise scores and all-cause and cardiovascular survival over 5 and 12 years are shown in figure 2. For comparison with pharmacological myocardial perfusion imaging (MPI) results obtained in a separate cohort of 1730 patients 
Table 1 Association of demographic, medication, resting vital sign and ECG variables, clinical diagnoses and arm exercise responses with all-cause and cardiovascular mortality over 5 and 12 years

\begin{tabular}{|c|c|c|c|c|c|c|}
\hline \multirow[b]{3}{*}{ Variable } & \multicolumn{6}{|c|}{ Arm exercise stress test $(\mathrm{N}=443)$} \\
\hline & \multicolumn{3}{|c|}{ 5-year } & \multicolumn{3}{|l|}{ 12-year } \\
\hline & $\begin{array}{l}\text { Survivors } \\
(n=323,72.9 \%)\end{array}$ & $\begin{array}{l}\text { All-cause mortality } \\
(n=120,27.1 \%)\end{array}$ & $\begin{array}{l}\text { CV mortality } \\
(n=50,11.3 \%)\end{array}$ & $\begin{array}{l}\text { Survivors } \\
(n=187,42.2 \%)\end{array}$ & $\begin{array}{l}\text { All-cause mortality } \\
(n=256,57.8 \%)\end{array}$ & $\begin{array}{l}\text { CV mortality } \\
(n=102,23.0 \%)\end{array}$ \\
\hline \multicolumn{7}{|l|}{ Demographics } \\
\hline \multirow[t]{2}{*}{ Age (years) (SD) } & $62.4(10.6)$ & $68.6(10.9)$ & $68.3(10.6)$ & $59.7(9.9)$ & $67.3(10.7)$ & $65.9(11.6)$ \\
\hline & & $p<0.001$ & $p<0.001$ & & $p<0.001$ & $p<0.001$ \\
\hline \multirow[t]{2}{*}{ Height (inches) (SD) } & $69.7(2.9)$ & $69.4(4.0)$ & $68.5(5.2)$ & $69.9(2.7)$ & $69.4(3.6)$ & $69.1(4.4)$ \\
\hline & & $p=0.36$ & $p=0.01$ & & $p=0.17$ & $p=0.08$ \\
\hline \multirow[t]{2}{*}{ Weight (lbs) (SD) } & $218.1(54.4)$ & $196.3(52.3)$ & $191.8(44.9)$ & $223.2(53.0)$ & $204.2(54.6)$ & $205.6(60.3)$ \\
\hline & & $p<0.001$ & $\mathrm{p}=0.001$ & & $\mathrm{p}<0.001$ & $\mathrm{p}=0.01$ \\
\hline \multirow[t]{2}{*}{ Body mass index $\left(\mathrm{kg} / \mathrm{m}^{2}\right)(\mathrm{SD})$} & $31.5(7.1)$ & $28.7(7.5)$ & $29.1(8.3)$ & $32.1(7.1)$ & $29.7(7.3)$ & $30.3(8.6)$ \\
\hline & & $p<0.001$ & $\mathrm{p}=0.03$ & & $p<0.001$ & $p=0.06$ \\
\hline \multirow[t]{2}{*}{ Race (black) (\%) } & $79(24.8)$ & $33(27.7)$ & $10(20.4)$ & $48(26.2)$ & $64(25.2)$ & $24(24)$ \\
\hline & & $p=0.54$ & $\mathrm{p}=0.50$ & & $\mathrm{p}=0.81$ & $\mathrm{p}=0.68$ \\
\hline \multirow[t]{2}{*}{ Gender (women) (\%) } & $8(2.5)$ & $1(0.8)$ & $1(2.0)$ & $5(2.7)$ & $4(1.6)$ & $3(2.9)$ \\
\hline & & $p=0.28$ & $p=0.84$ & & $p=0.41$ & $p=0.89$ \\
\hline \multicolumn{7}{|l|}{ Medications } \\
\hline \multirow[t]{2}{*}{$\beta$-blocker (\%) } & $125(38.7)$ & $55(45.8)$ & $30(60.0)$ & $75(40.1)$ & $105(41.0)$ & $47(46.1)$ \\
\hline & & $\mathrm{p}=0.17$ & $p=0.005$ & & $\mathrm{p}=0.85$ & $p=0.33$ \\
\hline \multirow[t]{2}{*}{ ACEI/ARB/hydralazine (\%) } & $168(52.0)$ & $66(55.0)$ & $31(62.0)$ & $91(48.7)$ & $143(55.9)$ & $63(61.8)$ \\
\hline & & $p=0.58$ & $\mathrm{p}=0.19$ & & $\mathrm{p}=0.13$ & $p=0.03$ \\
\hline \multirow[t]{2}{*}{ Statin (\%) } & $124(38.4)$ & $40(33.3)$ & $18(36.0)$ & $74(39.6)$ & $90(35.2)$ & $36(35.3)$ \\
\hline & & $p=0.33$ & $\mathrm{p}=0.75$ & & $p=0.34$ & $p=0.47$ \\
\hline \multirow[t]{2}{*}{ Platelet antagonist (\%) } & $190(58.8)$ & $63(52.5)$ & $28(56.0)$ & $100(53.5)$ & $153(59.8)$ & $60(58.8)$ \\
\hline & & $\mathrm{p}=0.23$ & $\mathrm{p}=0.71$ & & $p=0.19$ & $\mathrm{p}=0.38$ \\
\hline \multirow[t]{2}{*}{ Aldosterone blocker (\%) } & $13(4.0)$ & $4(3.3)$ & $0(0.0)$ & $6(3.2)$ & $11(4.3)$ & $1(1.0)$ \\
\hline & & $p=0.74$ & $\mathrm{p}=0.15$ & & $p=0.56$ & $p=0.24$ \\
\hline \multicolumn{7}{|l|}{ Resting vital signs } \\
\hline \multirow[t]{2}{*}{ Heart rate $(\mathrm{bpm})(\mathrm{SD})$} & $74(14)$ & $78(16)$ & $77(17)$ & $74(14)$ & $76(16)$ & $75(15)$ \\
\hline & & $p=0.01$ & $p=0.13$ & & $p=0.20$ & $p=0.55$ \\
\hline \multirow[t]{2}{*}{ Systolic BP (mm Hg) (SD) } & $136(21)$ & $132(23)$ & $132(22)$ & $135(20)$ & $135(22)$ & $135(23)$ \\
\hline & & $p=0.07$ & $p=0.23$ & & $p=0.80$ & $p=0.97$ \\
\hline \multirow[t]{2}{*}{ Diastolic BP (mm Hg) (SD) } & $83(12)$ & 78 (12) & 79 (13) & $84(12)$ & $80(13)$ & $80(13)$ \\
\hline & & $p<0.001$ & $p=0.03$ & & $p<0.001$ & $p=0.01$ \\
\hline \multicolumn{7}{|l|}{ Resting ECG results } \\
\hline \multirow[t]{2}{*}{ Abnormal (\%) } & $122(37.8)$ & $60(50.0)$ & $27(54.0)$ & $60(32.1)$ & $122(47.7)$ & $48(47.1)$ \\
\hline & & $p=0.02$ & $p=0.03$ & & $\mathrm{p}=0.001$ & $p=0.01$ \\
\hline \multicolumn{7}{|l|}{ Clinical diagnoses } \\
\hline \multirow[t]{2}{*}{ Hypertension (\%) } & $243(75.2)$ & $96(80.0)$ & $39(78.0)$ & $141(75.4)$ & $198(77.3)$ & $80(78.4)$ \\
\hline & & $\mathrm{p}=0.29$ & $\mathrm{p}=0.67$ & & $\mathrm{p}=0.63$ & $\mathrm{p}=0.56$ \\
\hline
\end{tabular}


Table 1 Continued

\begin{tabular}{|c|c|c|c|c|c|c|}
\hline \multirow[b]{3}{*}{ Variable } & \multicolumn{6}{|c|}{ Arm exercise stress test $(\mathrm{N}=443)$} \\
\hline & \multicolumn{3}{|c|}{ 5-year } & \multicolumn{3}{|l|}{ 12-year } \\
\hline & $\begin{array}{l}\text { Survivors } \\
(n=323,72.9 \%)\end{array}$ & $\begin{array}{l}\text { All-cause mortality } \\
(n=120,27.1 \%)\end{array}$ & $\begin{array}{l}\text { CV mortality } \\
(n=50,11.3 \%)\end{array}$ & $\begin{array}{l}\text { Survivors } \\
(n=187,42.2 \%)\end{array}$ & $\begin{array}{l}\text { All-cause mortality } \\
(n=256,57.8 \%)\end{array}$ & $\begin{array}{l}\text { CV mortality } \\
(n=102,23.0 \%)\end{array}$ \\
\hline \multirow[t]{2}{*}{ Dyslipidaemia (\%) } & $147(45.5)$ & $46(38.3)$ & $21(42.0)$ & $93(49.7)$ & $100(39.1)$ & $42(41.2)$ \\
\hline & & $p=0.18$ & $p=0.64$ & & $p=0.03$ & $p=0.16$ \\
\hline \multirow[t]{2}{*}{ Current smoker (\%) } & $100(31.0)$ & $44(36.7)$ & $20(40.0)$ & $54(28.9)$ & $90(35.2)$ & $39(38.2)$ \\
\hline & & $p=0.25$ & $p=0.20$ & & $p=0.16$ & $p=0.10$ \\
\hline \multirow[t]{2}{*}{ Past smoker (\%) } & $120(37.2)$ & $43(35.8)$ & $17(34.0)$ & $69(36.9)$ & $94(36.7)$ & $33(32.4)$ \\
\hline & & $p=0.80$ & $p=0.67$ & & $p=0.97$ & $p=0.44$ \\
\hline \multirow[t]{2}{*}{ Diabetes (\%) } & $111(34.4)$ & $50(41.7)$ & $20(40.0)$ & $58(31.0)$ & $103(40.3)$ & $39(38.2)$ \\
\hline & & $p=0.16$ & $p=0.44$ & & $\mathrm{p}=0.05$ & $p=0.21$ \\
\hline \multirow[t]{2}{*}{ CAD (\%) } & $126(39.0)$ & $56(46.7)$ & $29(58.0)$ & $69(36.9)$ & $113(44.1)$ & $51(50.0)$ \\
\hline & & $p=0.15$ & $p=0.01$ & & $p=0.13$ & $p=0.03$ \\
\hline \multirow[t]{2}{*}{ History of MI (\%) } & 87 (26.9) & $50(41.7)$ & $23(46.0)$ & $49(26.2)$ & $88(34.4)$ & $33(32.4)$ \\
\hline & & $p=0.003$ & $p=0.006$ & & $p=0.07$ & $p=0.27$ \\
\hline \multirow[t]{2}{*}{ History of CHF (\%) } & $29(9.0)$ & $28(23.3)$ & $17(34.0)$ & $11(5.9)$ & $46(18.0)$ & $24(23.5)$ \\
\hline & & $p<0.001$ & $p<0.001$ & & $p<0.001$ & $p<0.001$ \\
\hline \multirow[t]{2}{*}{ Status post CABG (\%) } & $53(16.4)$ & $29(24.2)$ & $17(34.0)$ & $27(14.4)$ & $55(21.5)$ & $29(28.4)$ \\
\hline & & $p=0.06$ & $p=0.003$ & & $p=0.06$ & $p=0.004$ \\
\hline \multirow[t]{2}{*}{ Status post $\mathrm{PCI}(\%)$} & 43 (13.3) & 16 (13.3) & $11(22.0)$ & $24(12.8)$ & 35 (13.7) & $20(19.6)$ \\
\hline & & $p=1.00$ & $p=0.10$ & & $p=0.80$ & $p=0.13$ \\
\hline \multirow[t]{2}{*}{ Atrial fibrillation (\%) } & $15(4.5)$ & $27(22.5)$ & $14(28.0)$ & $8(4.3)$ & 34 (13.3) & $16(15.7)$ \\
\hline & & $p<0.001$ & $p<0.001$ & & $p=0.001$ & $p<0.001$ \\
\hline \multirow[t]{2}{*}{ PAD (\%) } & $75(23.2)$ & $55(45.8)$ & $27(54.0)$ & $33(17.7)$ & 97 (37.9) & $44(43.1)$ \\
\hline & & $p<0.001$ & $p<0.001$ & & $p<0.001$ & $p<0.001$ \\
\hline \multirow[t]{2}{*}{ COPD (\%) } & $54(16.7)$ & $48(40.0)$ & $20(40.0)$ & $25(13.4)$ & $77(30.1)$ & $30(29.4)$ \\
\hline & & $p<0.001$ & $p<0.001$ & & $p<0.001$ & $p<0.001$ \\
\hline \multirow[t]{2}{*}{ Renal disease (\%) } & $19(5.9)$ & $18(15.0)$ & $8(16.0)$ & $9(4.8)$ & $28(10.9)$ & $10(9.8)$ \\
\hline & & $p=0.002$ & $p=0.01$ & & $p=0.02$ & $p=0.10$ \\
\hline \multirow[t]{2}{*}{ Cancer (\%) } & $20(6.2)$ & $26(21.7)$ & $7(14.0)$ & $5(2.7)$ & $41(16.0)$ & $10(9.8)$ \\
\hline & & $p<0.001$ & $p=0.04$ & & $p<0.001$ & $p=0.009$ \\
\hline \multicolumn{7}{|l|}{ Arm exercises responses } \\
\hline \multicolumn{7}{|l|}{ Peak values } \\
\hline \multirow[t]{2}{*}{ Heart rate $(\mathrm{bpm})(\mathrm{SD})$} & $125(21)$ & $117(25)$ & $115(26)$ & $129(20)$ & $119(23)$ & $117(22)$ \\
\hline & & $p<0.001$ & $p=0.002$ & & $p<0.001$ & $p<0.001$ \\
\hline \multirow[t]{2}{*}{ Systolic BP (mm Hg) (SD) } & $172(28)$ & $161(33)$ & $164(34)$ & $172(28)$ & $166(31)$ & $168(31)$ \\
\hline & & $p<0.001$ & $p=0.08$ & & $p=0.05$ & $p=0.29$ \\
\hline \multirow[t]{2}{*}{ Diastolic BP (mm Hg) (SD) } & $94(15)$ & 90 (17) & $91(17)$ & $95(15)$ & $91(16)$ & $92(16)$ \\
\hline & & $p=0.01$ & $p=0.28$ & & $p=0.02$ & $p=0.24$ \\
\hline \multicolumn{7}{|l|}{$\delta$ Values (Peak-Rest) } \\
\hline \multirow{2}{*}{ Heart rate $(\mathrm{bpm})(\mathrm{SD})$} & 52 (19) & $39(20)$ & 38 (19) & $56(19)$ & $43(20)$ & $42(18)$ \\
\hline & & $p<0.001$ & $p<0.001$ & & $p<0.001$ & $p<0.001$ \\
\hline
\end{tabular}




\begin{tabular}{|c|c|c|c|c|c|c|}
\hline \multirow[b]{3}{*}{ Variable } & \multicolumn{6}{|c|}{ Arm exercise stress test $(\mathrm{N}=443)$} \\
\hline & \multicolumn{3}{|c|}{ 5-year } & \multicolumn{3}{|l|}{ 12-year } \\
\hline & $\begin{array}{l}\text { Survivors } \\
(n=323,72.9 \%)\end{array}$ & $\begin{array}{l}\text { All-cause mortality } \\
(n=120,27.1 \%)\end{array}$ & $\begin{array}{l}\text { CV mortality } \\
(n=50,11.3 \%)\end{array}$ & $\begin{array}{l}\text { Survivors } \\
(n=187,42.2 \%)\end{array}$ & $\begin{array}{l}\text { All-cause mortality } \\
(n=256,57.8 \%)\end{array}$ & $\begin{array}{l}\text { CV mortality } \\
(n=102,23.0 \%)\end{array}$ \\
\hline \multirow[t]{2}{*}{ Systolic BP (mm Hg) (SD) } & $36(24)$ & $29(23)$ & $32(20)$ & $37(23)$ & $32(24)$ & $34(22)$ \\
\hline & $11(10)$ & $p=0.008$ & $p=0.31$ & & $p=0.03$ & $p=0.22$ \\
\hline \multirow[t]{2}{*}{ Diastolic BP (mm Hg) (SD) } & $11(12)$ & $12(12)$ & $13(11)$ & $11(14)$ & $12(11)$ & $13(12)$ \\
\hline & & $p=0.64$ & $p=0.54$ & & $p=0.45$ & $p=0.38$ \\
\hline \multicolumn{7}{|l|}{ Heart rate recovery (bpm) } \\
\hline \multirow[t]{2}{*}{1 min post (SD) } & $19(10)$ & $11(8)$ & $10(7)$ & $20(9)$ & $14(9)$ & $14(9)$ \\
\hline & & $p<0.001$ & $p<0.001$ & & $p<0.001$ & $p<0.001$ \\
\hline \multirow[t]{2}{*}{ Exercise capacity (METs) } & $3.3(0.9)$ & $2.6(0.8)$ & $2.6(0.8)$ & $3.5(0.9)$ & $2.8(0.9)$ & $2.8(0.9)$ \\
\hline & & $p<0.001$ & $p<0.001$ & & $p<0.001$ & $p<0.001$ \\
\hline \multicolumn{7}{|l|}{ Angina } \\
\hline \multirow[t]{2}{*}{ Limiting (\%) } & $7(2)$ & $2(2)$ & $2(4)$ & $3(2)$ & $6(2)$ & $2(2)$ \\
\hline & & $p=0.74$ & $p=0.43$ & & $p=0.59$ & $p=0.82$ \\
\hline \multirow[t]{2}{*}{ Non-limiting (\%) } & $17(6)$ & $11(10)$ & $8(16)$ & $9(5)$ & $19(8)$ & $11(11)$ \\
\hline & & $p=0.25$ & $p=0.03$ & & $p=0.59$ & $p=0.17$ \\
\hline \multirow[t]{2}{*}{ ST depression $\geq 1 \mathrm{~mm}(\%)$} & $39(13)$ & $23(20)$ & $12(26)$ & $19(10)$ & $43(18)$ & $21(21)$ \\
\hline & & $p=0.05$ & $p=0.02$ & & $p=0.03$ & $p=0.01$ \\
\hline \multirow[t]{2}{*}{ Ventricular dysrhythmias (\%) } & $26(8)$ & $17(14)$ & $11(22)$ & $8(4)$ & $35(14)$ & $16(16)$ \\
\hline & & $\mathrm{p}=0.05$ & $p=0.002$ & & $p=0.001$ & $p<0.001$ \\
\hline \multicolumn{7}{|c|}{$\begin{array}{l}\text { Data shown are means }(\mathrm{SD}) \text { or } \mathrm{n}(\%) \text {. } \\
\text { Statistically significant differences are designated in bold. } \\
\text { ACEI, ACE inhibitor; ARB, angiotensin receptor blocker; BP, blood pressure; CABG, coronary artery bypass grafting; CAD, coronary artery disease; CHF, congestive heart failure; COPD, chronic } \\
\text { obstructive pulmonary disease; CV, cardiovascular; METs, resting metabolic equivalents; MI, myocardial infarction; PAD, peripheral arterial disease; PCl, percutaneous coronary intervention. }\end{array}$} \\
\hline
\end{tabular}


Table 2 Multivariate HRs and $95 \%$ Cls for significant arm exercise variables by univariate analysis

\begin{tabular}{|c|c|c|c|c|c|}
\hline & $\begin{array}{l}\text { 5-year all-cause } \\
\text { mortality }\end{array}$ & 5-year CV mortality & $\begin{array}{l}\text { 5-year CV mortality } \\
\text { or MI }\end{array}$ & $\begin{array}{l}\text { 12-year all-cause } \\
\text { mortality }\end{array}$ & 12-year CV mortality \\
\hline Peak heart rate (bpm) & 1.00 (0.99 to 1.02$)$ & 1.00 (0.98 to 1.02$)$ & $1.00(0.98$ to 1.02$)$ & $1.00(0.99$ to 1.01$)$ & $1.00(0.98$ to 1.01$)$ \\
\hline Peak systolic blood pressure $(\mathrm{mm} \mathrm{Hg})$ & 0.99 (0.97 to 1.00$)$ & & & $1.00(0.99$ to 1.01$)$ & \\
\hline Peak diastolic blood pressure $(\mathrm{mm} \mathrm{Hg})$ & 1.01 (0.99 to 1.02$)$ & & & 0.99 (0.98 to 1.01$)$ & \\
\hline$\delta$ Heart rate $(\mathrm{bpm})$ & 1.01 (0.97 to 1.03$)$ & 0.99 (0.95 to 1.02$)$ & $1.00(0.97$ to 1.03$)$ & 1.00 (0.99 to 1.02$)$ & 0.99 (0.97 to 1.02$)$ \\
\hline$\delta$ Systolic blood pressure $(\mathrm{mm} \mathrm{Hg})$ & 1.01 (0.99 to 1.02$)$ & & & $1.00(0.99$ to 1.01$)$ & \\
\hline Heart rate recovery (bpm) & $0.93^{\star \star \star}(0.90$ to 0.96$)$ & $0.92^{\star \star}(0.86$ to 0.97$)$ & $0.93^{\star *}(0.89$ to 0.98$)$ & $0.96^{\star \star \star}(0.94$ to 0.98$)$ & $0.96^{\star}(0.92$ to 1.00$)$ \\
\hline Exercise capacity (METs) & $0.44^{\star * \star}(0.30$ to 0.65$)$ & $0.55^{\star}(0.32$ to 0.95$)$ & $0.51^{\star \star}(0.32$ to 0.81$)$ & $0.55^{\star \star \star}(0.43$ to 0.70$)$ & $0.55^{\star \star \star}(0.38$ to 0.79$)$ \\
\hline Non-limiting Angina & & $1.60(0.34$ to 7.51$)$ & & & \\
\hline ST depression $\geq 1 \mathrm{~mm}$ & $1.85^{\star}(1.00$ to 3.43$)$ & $2.59^{\star}(1.00$ to 6.71$)$ & $3.07^{\star *}(1.44$ to 6.57$)$ & $1.70^{*}(1.10$ to 2.61$)$ & $2.48^{\star \star}(1.34$ to 4.59$)$ \\
\hline Ventricular dysrhythmias & & $2.08(0.88$ to 4.96$)$ & $1.90(0.90$ to 4.02$)$ & $1.47(0.94$ to 2.31$)$ & $1.38(0.69$ to 2.79$)$ \\
\hline
\end{tabular}

Table 3 Multivariate parameter estimates, HRs and 95\% Cls for arm exercise variables based on step-wise Cox analysis

\begin{tabular}{|c|c|c|c|c|c|c|c|c|c|c|}
\hline & \multicolumn{2}{|c|}{ 5-year all-cause mortality } & \multicolumn{2}{|c|}{ 5-year CV mortality } & \multicolumn{2}{|c|}{ 5-year CV mortality or MI } & \multicolumn{2}{|c|}{$\begin{array}{l}\text { 12-year all-cause } \\
\text { mortality }\end{array}$} & \multicolumn{2}{|c|}{ 12-year CV mortality } \\
\hline & $\begin{array}{l}\text { Parameter } \\
\text { estimate }\end{array}$ & HR & $\begin{array}{l}\text { Parameter } \\
\text { estimate }\end{array}$ & HR & $\begin{array}{l}\text { Parameter } \\
\text { estimate }\end{array}$ & HR & $\begin{array}{l}\text { Parameter } \\
\text { estimate }\end{array}$ & HR & $\begin{array}{l}\text { Parameter } \\
\text { estimate }\end{array}$ & HR \\
\hline $\begin{array}{l}\text { Heart rate recovery } \\
\text { (bpm) }\end{array}$ & -0.06 & $\begin{array}{l}0.94^{* * *} \\
(0.91 \text { to } 0.97)\end{array}$ & -0.10 & $\begin{array}{l}0.90^{\star * *} \\
(0.86 \text { to } 0.95)\end{array}$ & -0.08 & $\begin{array}{l}0.93^{\star * \star} \\
(0.89 \text { to } 0.96)\end{array}$ & -0.04 & $\begin{array}{l}0.96^{\star * \star} \\
(0.94 \text { to } 0.98)\end{array}$ & -0.05 & $\begin{array}{l}0.95^{\star *} \\
(0.92 \text { to } 0.98)\end{array}$ \\
\hline $\begin{array}{l}\text { Exercise capacity } \\
\text { (METs) }\end{array}$ & -0.78 & $\begin{array}{l}0.46^{* \star *} \\
(0.33 \text { to } 0.63)\end{array}$ & -0.75 & $\begin{array}{l}0.47^{\star \star} \\
(0.29 \text { to } 0.77)\end{array}$ & -0.73 & $\begin{array}{l}0.48^{\star \star \star} \\
(0.32 \text { to } 0.73)\end{array}$ & -0.63 & $\begin{array}{l}0.54^{\star \star \star} \\
(0.44 \text { to } 0.65)\end{array}$ & -0.72 & $\begin{array}{l}0.49^{\star \star \star} \\
(0.36 \text { to } 0.67)\end{array}$ \\
\hline ST depression $\geq 1 \mathrm{~mm}$ & 0.76 & $\begin{array}{l}2.14^{\star *} \\
(1.22 \text { to } 3.78)\end{array}$ & 1.07 & $\begin{array}{l}2.92^{\star \star} \\
(1.33 \text { to } 6.41)\end{array}$ & 1.22 & $\begin{array}{l}3.38^{\star \star \star} \\
(1.70 \text { to } 6.70)\end{array}$ & 0.61 & $\begin{array}{l}1.85^{\star *} \\
(1.24 \text { to } 2.76)\end{array}$ & 0.90 & $\begin{array}{l}2.46^{\star \star} \\
(1.37 \text { to } 4.42)\end{array}$ \\
\hline
\end{tabular}


Table 4 Multivariate HRs and $95 \%$ Cls for arm exercise scores and significant covariates by univariate analysis

\begin{tabular}{|c|c|c|c|c|c|}
\hline \multirow[t]{2}{*}{ Variable } & \multicolumn{5}{|l|}{ Outcome } \\
\hline & 5-year all-cause mortality & 5-year CV mortality & 5-year CV mortality or MI & 12-year all-cause mortality & 12-year CV mortality \\
\hline Arm exercise score & $0.96^{\star \star \star}(0.94$ to 0.98$)$ & $0.92^{\star \star \star}(0.88$ to 0.96$)$ & $0.95^{\star \star \star}(0.92$ to 0.97$)$ & $0.96^{\star \star \star}(0.95$ to 0.98$)$ & $0.96^{\star \star \star}(0.94$ to 0.98$)$ \\
\hline \multicolumn{6}{|l|}{ Demographics } \\
\hline Age (years) & 1.01 (0.99 to 1.04$)$ & 0.99 (0.96 to 1.03$)$ & $1.01(0.98$ to 1.04$)$ & $1.02(1.00$ to 1.03$)$ & 1.01 (0.99 to 1.04$)$ \\
\hline Body mass index $\left(\mathrm{kg} / \mathrm{m}^{2}\right)$ & $0.96^{*}(0.92$ to 0.99$)$ & 0.98 (0.93 to 1.04$)$ & & $0.97^{\star \star}(0.95$ to 0.99$)$ & 0.99 (0.96 to 1.02$)$ \\
\hline \multicolumn{6}{|l|}{ Medications } \\
\hline$\beta$-blocker & & 0.92 (0.43 to 1.96$)$ & & & \\
\hline ACEI/ARB/hydralazine & & & & & $1.43(0.87$ to 2.34$)$ \\
\hline \multicolumn{6}{|l|}{ Resting vital signs } \\
\hline Heart rate $(\mathrm{bpm})$ & $1.02(1.00$ to 1.03$)$ & & & & \\
\hline Diastolic BP $(\mathrm{mm} \mathrm{Hg})$ & $0.98^{\star}(0.96$ to 1.00$)$ & $1.00(0.97$ to 1.03$)$ & & $0.99^{\star}(0.97$ to 1.00$)$ & 1.00 (0.98 to 1.02$)$ \\
\hline \multicolumn{6}{|l|}{ Resting ECG results } \\
\hline Abnormal & $1.03(0.63$ to 1.70$)$ & $0.54(0.25$ to 1.14$)$ & $0.73(0.40$ to 1.36$)$ & $1.18(0.86$ to 1.61$)$ & 0.93 (0.56 to 1.55$)$ \\
\hline \multicolumn{6}{|l|}{ Clinical diagnoses } \\
\hline Dyslipidaemia & & & & $0.67^{\star \star}(0.49$ to 0.90$)$ & \\
\hline Diabetes & & & & $0.91(0.66$ to 1.26$)$ & \\
\hline CAD & & 1.40 (0.57 to 3.46$)$ & & & 0.97 (0.53 to 1.77$)$ \\
\hline History of MI & 1.29 (0.81 to 2.05$)$ & $1.24(0.55$ to 2.78$)$ & $1.46(0.79$ to 2.68$)$ & & \\
\hline History of $\mathrm{CHF}$ & 1.17 (0.64 to 2.13$)$ & 1.61 (0.67 to 3.86$)$ & $1.96(1.00$ to 3.84$)$ & $1.43(0.94$ to 2.19$)$ & $1.63(0.84$ to 3.16$)$ \\
\hline Status post $C A B G$ & & $1.71(0.71$ to 4.10$)$ & 1.22 (0.61 to 2.43$)$ & & 1.53 (0.81 to 2.89$)$ \\
\hline Atrial fibrillation & $3.50^{\star \star \star}(1.92$ to 6.38$)$ & $6.83^{\star \star \star}(2.77$ to 16.88$)$ & $4.31^{\star \star \star}(2.03$ to 9.16$)$ & $2.09^{\star \star}(1.28$ to 3.39$)$ & $3.27^{\star \star}(1.47$ to 7.25$)$ \\
\hline PAD & $1.75^{\star}(1.07$ to 2.84$)$ & $1.73(0.75$ to 3.94$)$ & $2.44^{\star \star}(1.29$ to 4.61$)$ & $1.74^{\star \star}(1.24$ to 2.43$)$ & $2.04^{\star *}(1.19$ to 3.47$)$ \\
\hline COPD & $1.19(0.73$ to 1.95$)$ & 1.06 (0.47 to 2.37$)$ & $1.12(0.60$ to 2.09$)$ & $1.26(0.91$ to 1.77$)$ & 1.41 (0.81 to 2.45$)$ \\
\hline Renal disease & $1.82(0.87$ to 3.81$)$ & 2.61 (0.98 to 6.94$)$ & 1.74 (0.75 to 4.05$)$ & $1.30(0.75$ to 2.24$)$ & \\
\hline Cancer & $2.41^{\star \star}(1.28$ to 4.55$)$ & 1.64 (0.44 to 6.08$)$ & & $2.17^{\star \star}(1.35$ to 3.49$)$ & 1.07 (0.36 to 3.17$)$ \\
\hline
\end{tabular}



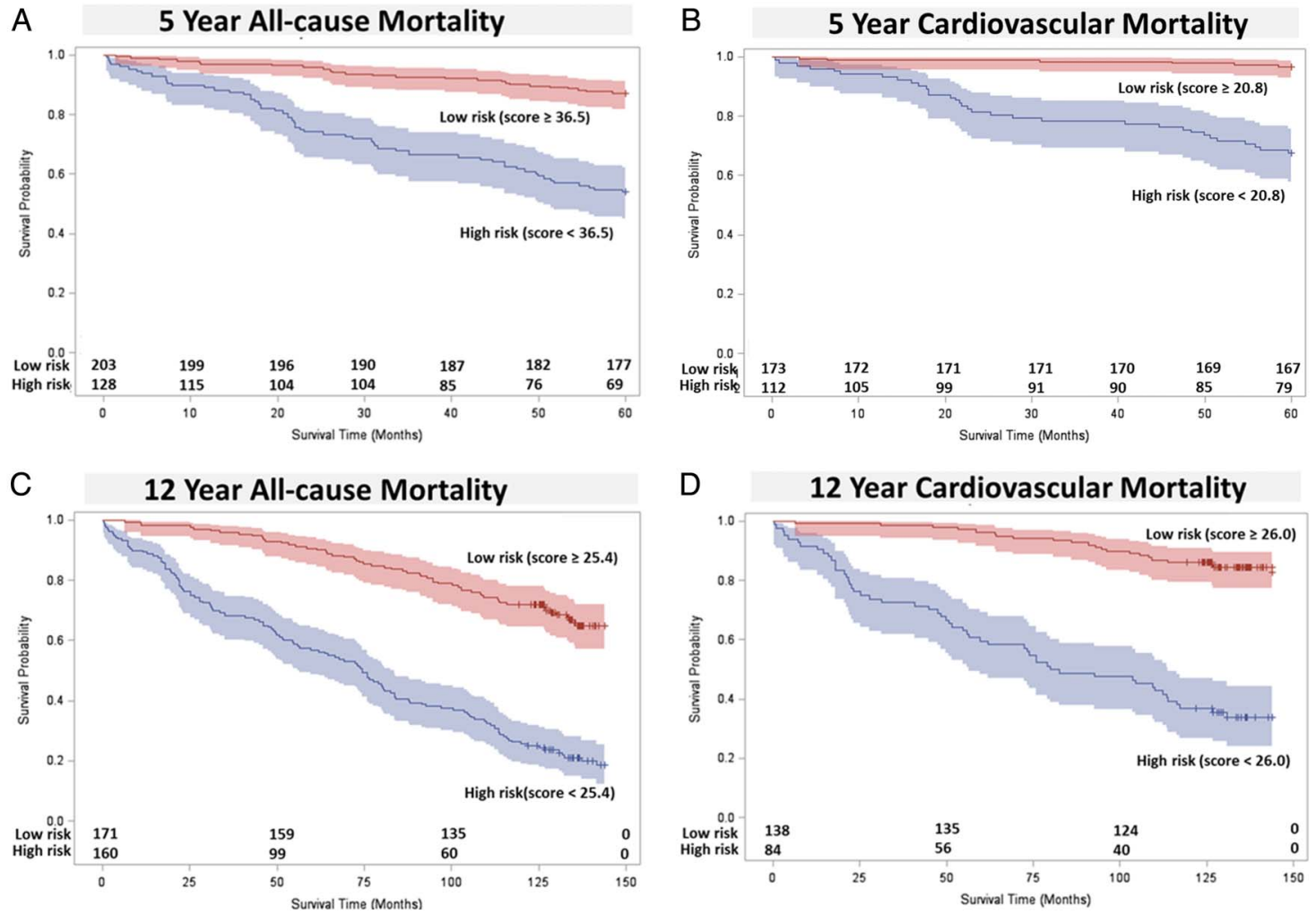

Figure 1 Kaplan-Meier plots of 5 and 12-year all cause (A and C, respectively) and cardiovascular mortality (B and D, respectively) with number of participants at risk and $95 \%$ Cls stratified by cut points into high and low-risk groups based on arm exercise scores.

evaluated over the same 1997-2002 time interval, ${ }^{7}$ receiver operator curve plots of arm exercise scores, best fit models of significant pharmacologic MPI study 5-year and 12-year mortality predictors (perfusion defect number, type, and size and the change in heart rate from baseline to peak stress as described recently $^{7}$ ), and an abnormal versus normal pharmacological MPI result, are depicted in figure 3 .

\section{DISCUSSION}

For the $\geq 50 \%$ of patients unable to perform treadmill or other leg exercise, we have developed a scoring system for prediction of all-cause and cardiovascular mortality over 5 and 12 years and cardiovascular mortality or MI over 5 years using easily obtainable information from arm exercise ECG stress testing. No other scoring system and few prognostic data are currently available for arm exercise testing. However, we have recently observed that arm exercise is an equivalent or superior alternative to pharmacological MPI for many patients who are unable to perform lower extremity exercise. ${ }^{7}$ As illustrated in figure 3, this is further emphasised by the higher area under the curve of C-statistic plots for prediction of mortality with arm exercise ECG stress test scores than for pharmacological MPI results. Arm exercise also provides important functional and physiological information on exercise capacity, heart rate recovery and

Table 5 Arm exercise scores with $\mathrm{C}$ statistic values before and after adjustment for significant demographic and clinical predictors

\begin{tabular}{|c|c|c|c|}
\hline \multirow[b]{2}{*}{ Outcome } & \multirow[b]{2}{*}{ Score equation } & \multicolumn{2}{|c|}{ Area under the curve (AUC) ${ }^{*}$} \\
\hline & & Unadjusted & Adjusted \\
\hline 5-year-all-cause mortality & $\mathrm{HRR}+13.0 \times \mathrm{METs}-12.6 \times \mathrm{ST} \Delta(0 / 1)$ & 0.77 & 0.86 \\
\hline 5-year-CV mortality & $\mathrm{HRR}+7.3 \times \mathrm{METs}-10.5 \times \mathrm{ST} \Delta(0 / 1)$ & 0.81 & 0.88 \\
\hline 5-year-CV mortality or MI & $\mathrm{HRR}+9.4 \times \mathrm{METs}-15.7 \times \mathrm{ST} \Delta(0 / 1)$ & 0.79 & 0.83 \\
\hline 12-year-all-cause mortality & $\mathrm{HRR}+14.5 \times \mathrm{METs}-14.2 \times \mathrm{ST} \Delta(0 / 1)$ & 0.77 & 0.83 \\
\hline 12-year-CV mortality & $\mathrm{HRR}+13.8 \times \mathrm{METs}-17.1 \times \mathrm{ST} \Delta(0 / 1)$ & 0.78 & 0.82 \\
\hline
\end{tabular}

${ }^{*}$ C-statistic (AUC) based on a logistic model.

†Arm Exercise Score adjusted for significant demographic and clinical variables indicated in table 4.

AUC, area under the curve; CV, cardiovascular; HRR, heart rate recovery at 1 min postexercise; METs, resting metabolic equivalents; MI, myocardial infarction; ST, ST segment depression. 
A

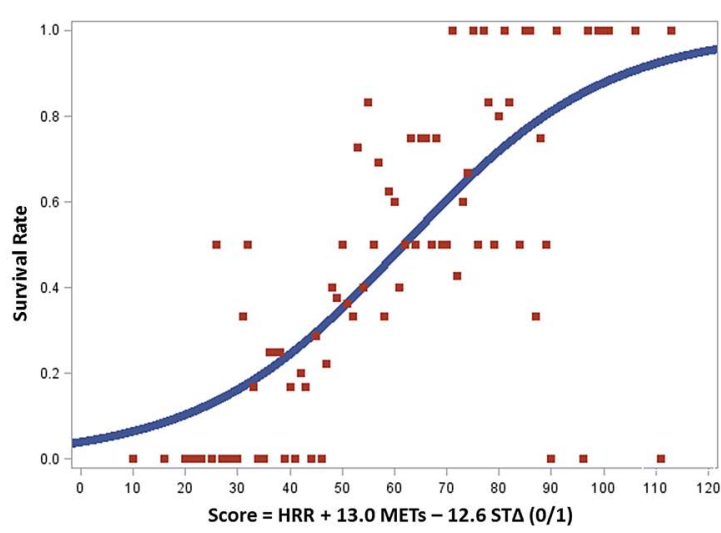

C

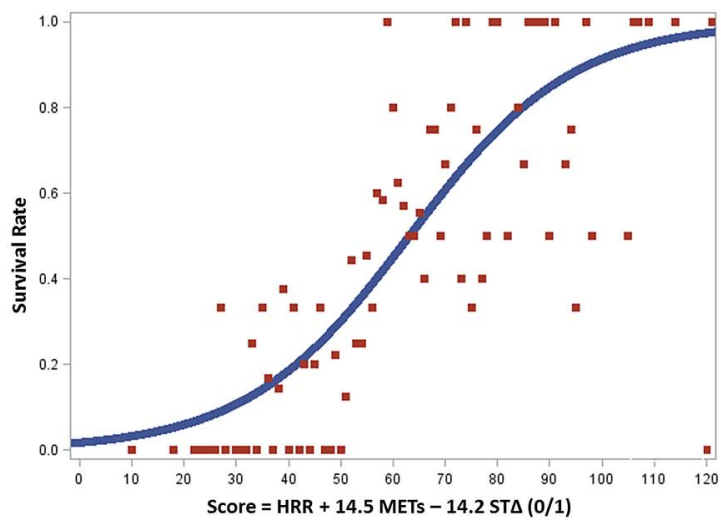

B

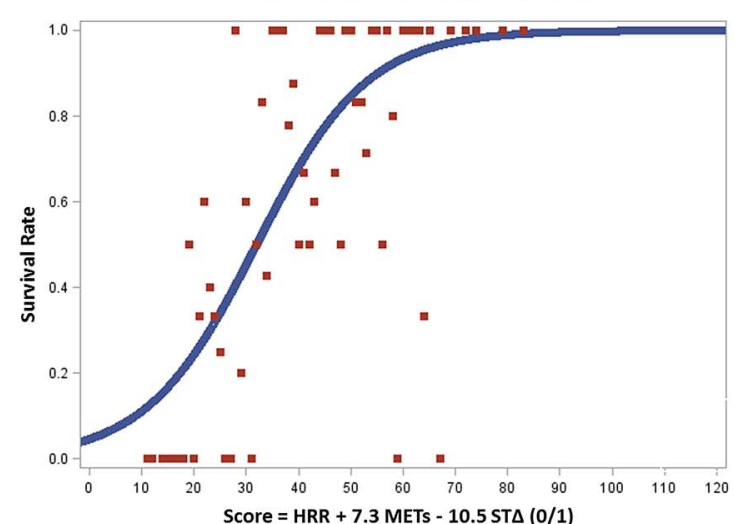

D

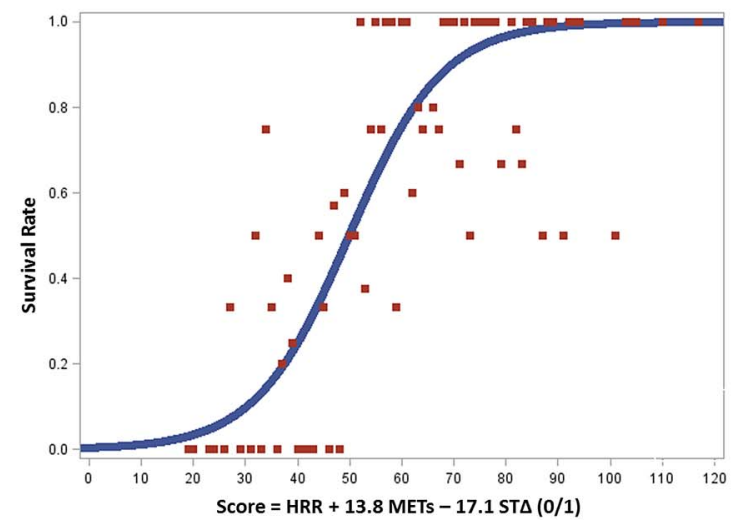

Figure 2 The continuous curvilinear relationship between arm exercise scores and 5-year and 12-year all-cause (A and C, respectively) and cardiovascular survival ( $B$ and $D$, respectively) with associated scatter plots. HRR, heart rate recovery at 1-min postexercise; METs, arm exercise capacity in resting metabolic equivalents; ST $\Delta$, ST segment depression either $<1 \mathrm{~mm}(0)$ or $\geq 1 \mathrm{~mm}$ (1).

clinical symptoms relevant to patient management decisions such as referral for cardiac catheterisation. This information is not available with pharmacological MPI or echocardiographic evaluations. Although arm and leg exercise ECG stress tests are less sensitive for detection of cardiac ischaemia than MPI, ${ }^{7} 10{ }^{11}$ we have demonstrated a close relationship between an abnormal arm exercise ECG result and the size and reversibility of perfusion defects. ${ }^{10}{ }^{15}$ Arm exercise ECG testing does not require exposure to ionising radiation and is several times less expensive and time consuming for patients and staff than pharmacological imaging. Institutional resource and space requirements are also much less for arm exercise ECG testing than for treadmill or pharmacological imaging evaluations.

We observed a C-statistic of 0.81 for prediction of cardiovascular mortality over 5 years with arm exercise, which is similar to C-statistic results of 0.85 and 0.75 in outpatients and inpatients, respectively, for prognostication of cardiovascular mortality over 4-5 years with the Duke Treadmill Score. ${ }^{12}$ However, despite the ability of arm exercise and Duke Treadmill Score C-statistics to discriminate outcome events, there are notable differences between our cohort and that from which the Duke
Treadmill Score was derived. Our cohort is comprised of a very high-risk veteran population with a 5 -year cardiovascular mortality of about $11 \%$ in comparison with the 3-4\% 4-year cardiovascular mortality for outpatients and $8 \%$ mortality for inpatients in the Duke sample. ${ }^{12}$ The high mortality of our population is typical of patients unable to perform treadmill exercise. ${ }^{4}$ Our cohort included inpatients and outpatients, was about 10 15 years older, and was comprised of $98 \%$ men versus $67-70 \%$ men in the Duke database. ${ }^{12}$ The prevalence of peripheral arterial disease, congestive heart failure and atrial fibrillation in our population was 29\%, 13\%, and $10 \%$, respectively, all of which were associated with worsened cardiovascular mortality. The greater relative prognostic value of clinical predictors in our population versus the cohort used to derive the Duke Treadmill Score $^{2}$ may reflect the much higher outcome event rate in our group. However, functional assessment and estimation of prognosis in these high-risk patients is likely to be easier with arm exercise scores than with more complex models of clinical variables, despite their comparable C-statistic values.

In contrast to the Duke Treadmill Score, which stratified patients into high, low and intermediate-risk 
A

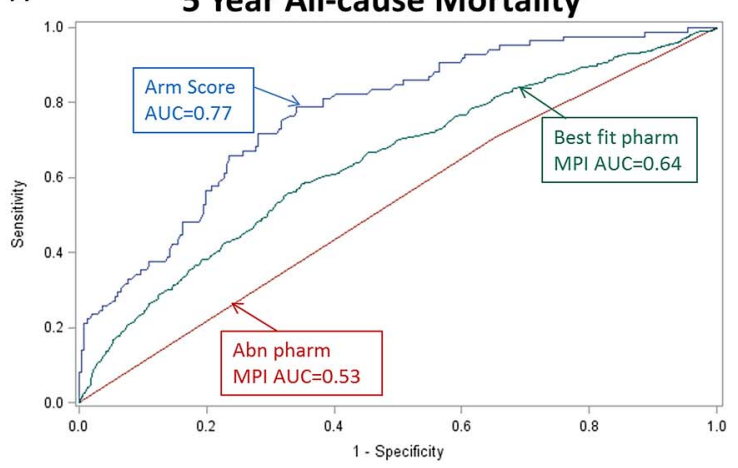

C

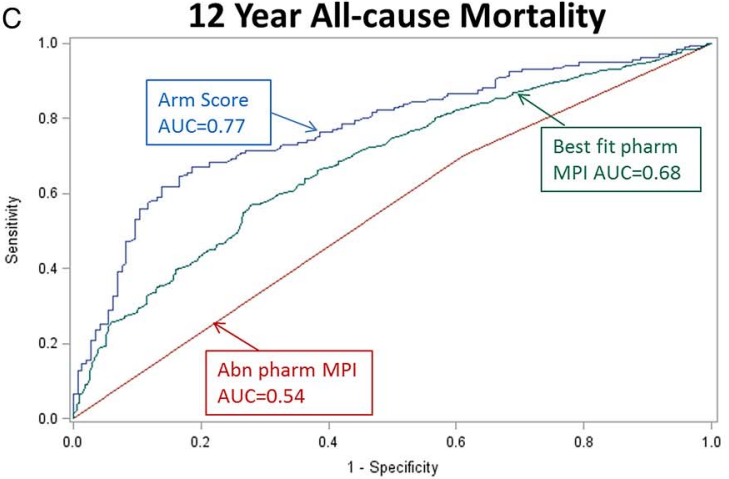

B

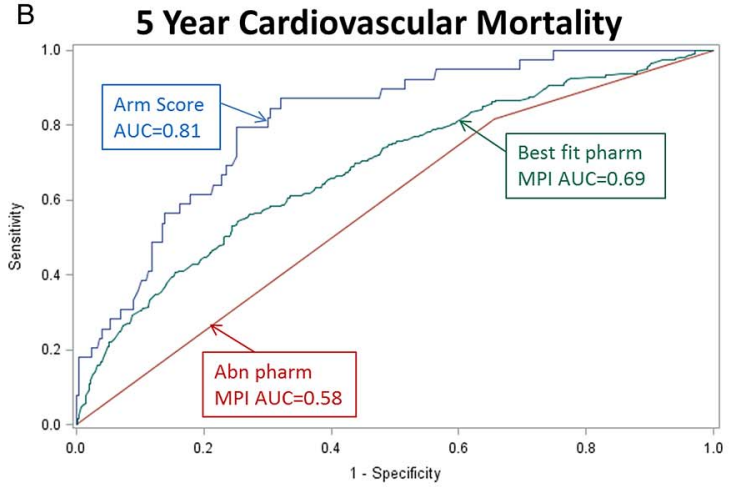

D

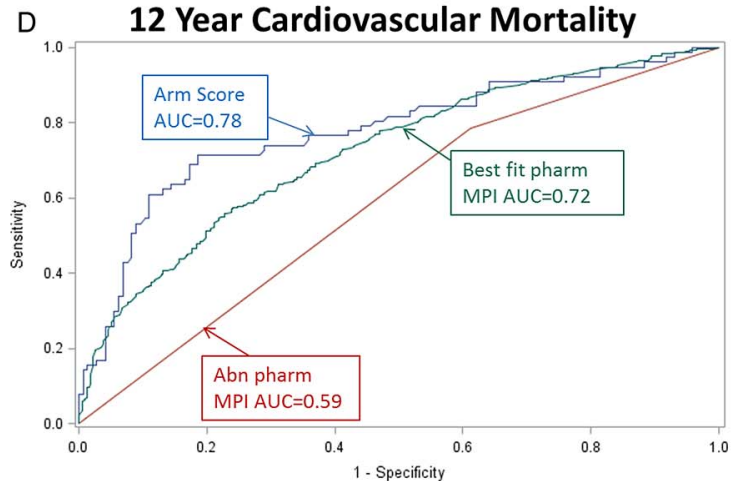

Figure 3 Receiver operator curve plots for prognostication of 5-year and 12-year all-cause (A and C, respectively) and cardiovascular mortality (B and D, respectively) with arm exercise scores (Arm Score AUC), best fit model pharmacological myocardial perfusion imaging (MPI) study predictors (perfusion defect number, type and size and the change in heart rate from rest to peak stress; ${ }^{7}$ Best fit pharm MPI AUC), and an abnormal versus normal pharmacological MPI result (Abn pharm MPI AUC), for 1730 pharmacological evaluations performed during the same 1997-2002 time interval. AUC, area under the curve.

groups, ${ }^{2}$ best fit statistical models of our arm exercise cohort identified only very high and relatively low-risk groups. The difference in 5-year cardiovascular mortality between the higher and lower risk groups in our cohort (29\% vs $3 \%$ ) was similar to the Duke sample over 45 years $(28 \%$ vs $1-2 \%) .^{2}$ However, we did not identify an intermediate risk group, which comprised about half of the Duke sample, ${ }^{2}$ and the proportion of patients in the high-risk group of our cohort was much greater than in the Duke cohort $(39 \%$ vs $8 \%) .^{2}$ This difference is likely related to the very high-risk nature of our population, which experienced a 5-year all-cause mortality rate of $23 \%$. Rates of all-cause and cardiovascular survival in our cohort may be estimated over a continuum of arm exercise scores based on curvilinear relationships shown in figure 2.

Our arm ergometer score and the Duke Treadmill Score are comprised of three test variables that include exercise capacity and stress-induced ST segment depression data. ${ }^{2}$ However, there are other important differences in the individual components. First, the Duke Treadmill Score does not include heart rate recovery, which is an element of our arm exercise score, but was not found to be strongly associated with mortality until several years after publication of the Duke results. ${ }^{2} 5$ Second, we did not observe an association of angina, either limiting or non-limiting, with cardiovascular mortality, whereas this finding is a component of the Duke Treadmill Score. ${ }^{2}$ One explanation for this is that unlike the Duke investigators, we did not censor our results for coronary revascularisation, which has become much more common than during the 1969-1990 era when the Duke data were obtained. ${ }^{1}{ }^{2}$ We have previously observed that arm exercise-induced angina was strongly associated with subsequent coronary revascularisation, ${ }^{9}$ which also predicted lower mortality in our population, possibly mitigating the effect of angina as a prognosticator of death. Thus, our data reflect actual clinical practice outcomes rather than the natural history of coronary artery disease. In addition, certain classes of medications such as statins and platelet antagonists, which also improve cardiovascular mortality, were not widely prescribed before 1990, and might influence the natural history of patients with angina. However, there was no association of medications with mortality in our cohort. Finally, in contrast to the Duke Treadmill Score, ${ }^{2}$ we did not observe a relationship between the magnitude of arm exercise-induced ST segment depression and subsequent cardiovascular mortality. This difference could be explained by our smaller sample size or by the effects of subsequent coronary revascularisation and/or medical treatment on cardiovascular mortality of patients with a 
marked arm exercise-induced ST segment depression as noted above for individuals with stress-induced angina. In this context, we have also observed an association between arm exercise-induced ST-segment depression and coronary revascularisation. ${ }^{9}$

Arm exercise capacity in METs and heart rate recovery exhibited robust inverse relationships with cardiovascular, non-cardiovascular and all-cause mortality after adjustment for covariates and there was no interaction of these effects with coronary revascularisation. These results are similar to findings with treadmill or leg cycle ergometer testing, for which several investigators have reported powerful inverse relationships between exercise capacity and/or heart rate recovery and long term all-cause and cardiovascular mortality in lower risk populations. ${ }^{5} 12$ 1618 Our arm exercise score and pharmacological MPI results are also consistent with other studies demonstrating that leg exercise capacity is a better predictor of mortality than stress nuclear perfusion imaging results. ${ }^{18} 19$

There are important differences in cardiovascular responses to arm and leg exercise, however. In young healthy participants, peak oxygen uptake with arm exercise averaged $56 \%$ of treadmill maximal oxygen uptake. ${ }^{8}$ Peak heart rate was 5-8\% lower for arm versus leg work whereas systolic blood pressure is greater for arm exercise. $^{8}{ }^{20}$ Thus, the rate-pressure product and cardiac work requirement are comparable for the two exercise modalities, despite the lower peak oxygen uptake during arm exercise.

Since our study was initiated in 1997, new imaging modalities such as coronary CT angiography have emerged $^{21}$ and the number of patients unable to perform treadmill exercise continues to increase. ${ }^{4}$ These new imaging modalities provide potentially useful information but, similar to MPI stress testing, require exposure to ionising radiation, ${ }^{21}{ }^{22}$ contribute to rapidly increasing healthcare costs, ${ }^{23}$ and fail to provide prognostic and functional information on exercise capacity, symptomatic, cardiovascular and ECG responses to the relevant physiological stress of exercise. Nephrotoxicity also is a potential limitation of coronary CT angiography. Arm exercise ECG stress testing is a potential solution to these problems for many patients unable to perform leg exercise.

Major limitations of this arm exercise scoring system are that it is based on retrospective data and was developed in a high-risk cohort of veterans, possibly introducing selection bias. Definitive confirmation of this approach will require a randomised prospective clinical trial of arm exercise versus pharmacologic stress testing, which is being planned. Generalisability of these equations to younger, healthier populations or to women is uncertain and also will require further investigation. Arm exercise is not feasible for patients with upper extremity disabilities and ECG testing alone may not be adequate for patients with left bundle branch block or other ECG abnormalities that interfere with accurate interpretation of the stress ECG.
In conclusion, we have developed a simple arm exercise ECG scoring system for prediction of 5-year and 12-year all-cause and cardiovascular mortality and 5-year cardiovascular mortality or MI. These arm exercise scores have comparable accuracy to the Duke Treadmill Score, are at least equivalent to pharmacological MPI for this purpose, and are the only data available for patients who cannot perform leg exercise. Thus, greater clinical use of arm exercise ECG stress testing in this population is a potentially equivalent or superior alternative to non-selective pharmacological imaging for cost-effective clinical decision making without radiation exposure.

Contributors WM designed and supervised the study and data collection, obtained grant funding, wrote the manuscript and is responsible for its overall content. PC and EB performed data extraction, analysis, and interpretation, and assisted with manuscript preparation. $\mathrm{YX}, \mathrm{HX}$ and LW were responsible for statistical analyses, tables and graphs, provided intellectual contributions, and assisted with manuscript preparation.

Funding HX, PC, and WHM and EB are supported by a Merit Review research award from the Department of Veterans Affairs, Washington DC.

Competing interests None declared.

Ethics approval St. Louis Veterans Administration Institutional Review Board.

Provenance and peer review Not commissioned; externally peer reviewed.

Data sharing statement All data that are currently available from this study have either been published or are contained in the submitted manuscript.

Open Access This is an Open Access article distributed in accordance with the Creative Commons Attribution Non Commercial (CC BY-NC 4.0) license, which permits others to distribute, remix, adapt, build upon this work noncommercially, and license their derivative works on different terms, provided the original work is properly cited and the use is non-commercial. See: http:// creativecommons.org/licenses/by-nc/4.0/

\section{REFERENCES}

1. Mark DB, Hlatky MA, Harrell FE Jr, et al. Exercise treadmill score for predicting prognosis in coronary artery disease. Ann Intern Med 1987;106:793-800.

2. Mark DB, Shaw L, Harrell FE Jr, et al. Prognostic value of a treadmill exercise score in outpatients with suspected coronary artery disease. N Eng J Med 1991;325:849-53.

3. Nishime EO, Cole CR, Blackstone $\mathrm{EH}$, et al. Heart rate recovery and treadmill exercise score as predictors of mortality in patients referred for exercise ECG. JAMA 2000;284:1392-8.

4. Rozanski A, Gransar H, Hayes SW, et al. Temporal trends in the frequency of inducible myocardial ischemia during cardiac stress testing: 1991 to 2009. J Am Coll Cardiol 2013;61:1054-65.

5. Cole CR, Blackstone EH, Pashkow FJ, et al. Heart rate recovery immediately after exercise as a predictor of mortality. $N$ Eng J Med 1999;341:1351-7.

6. Shetler K, Marcus R, Froelicher VF, et al. Heart rate recovery validation and methodologic issues. J Am Coll Cardiol 2001;38:1980-7.

7. Martin WH III, Xian H, Chandiramani $\mathrm{P}$, et al. Cardiovascular mortality prediction in veterans with arm exercise vs pharmacologic myocardial perfusion imaging. Am Heart J 2015;170:362-370e2.

8. Ilias NA, Xian $\mathrm{H}$, Inman $\mathrm{C}$, et al. Arm exercise testing predicts clinical outcome. Am Heart J 2009;157:69-76.

9. Martin WH III, Xian H, Wagner D, et al. Arm exercise as an alternative to pharmacologic stress testing: arm exercise stress testing and outcome. Am Heart J 2014;167:169-77.

10. Chan AK, Ilias-Khan NA, Xian $\mathrm{H}$, et al. Arm exercise stress perfusion imaging predicts clinical outcome. J Appl Physiol 2011;111:1546-53.

11. Chaitman BR. Exercise stress testing. In: Zipes DP, Libby P, Bonow $\mathrm{RO}$, et al., eds. Braunwald's heart disease: a textbook of 
cardiovascular medicine. Vol 1. 7th edn. Philadelphia, PA: Elsevier Saunders, 2005:153-85.

12. Myers J, Prakash M, Froelicher V, et al. Exercise capacity and mortality among men referred for exercise testing. $N$ Eng $\mathrm{J}$ Med 2002;346:793-801.

13. Astrand PO, Rodahl K. Textbook of work physiology. 3rd edn. New York: McGraw-Hill, 1986:365. Ch. 8.

14. SAS software. Version 9.4 of the SAS System for Windows; [last accessed 9/15/2014]; copyright 2014. SAS Institute, Inc. Cary, NC, USA.

15. Martin WH III, Xian H, Wagner DL, et al. Arm exercise myocardial perfusion imaging for prognostication of long-term outcome. Med Sci Sports Exerc 2014;46:2216-23.

16. Blair SN, Kohl HW III, Paffenbarger RS Jr, et al. Physical fitness and all-cause mortality: a prospective study of healthy men and women. JAMA 1989;262:2395-401.

17. Jouven X, Empana JP, Schwartz PJ, et al. Heart rate profile during exercise as a predictor of sudden death. $N$ Eng $J$ Med 2005;352:1951-8.

18. Snader CF, Marwick TH, Pashkow FJ, et al. Importance of estimated functional capacity as a predictor of all-cause mortality among patients referred for exercise thallium single-photon emission computed tomography: a report of 3400 patients from a single center. J Am Coll Cardiol 1997;30:641-8.

19. Candell-Riera J, Ferreira-Gonzalez I, Marsal JR, et al. Usefulness of exercise test and myocardial perfusion-gated single photon emission computed tomography to improve prediction of major events. Circ Cardiovasc Imaging 2013;6:531-4.

20. Stenberg J, Astrand PO, Ekblom B, et al. Hemodynamic response to work with different muscle groups, sitting and supine. J Appl Physiol 1967;22:61-70.

21. Douglas PS, Hoffmann L, Patel MR, et al., for the PROMISE Investigators. Outcomes of anatomical versus functional testing for coronary artery disease. N Eng J Med 2015;372: 1291-300.

22. Berrington de Gonzalez A, Kim KP, Smith-Bindman R, et al. Myocardial perfusion scans: projected population cancer risks from current levels of use in the United States. Circulation 2010;122:2403-10.

23. Fuchs VR, Milstein A. The $\$ 640$ billion question-why does cost-effective care diffuse so slowly? N Eng J Med 2011;364:1985-7. 